Research Paper

\title{
Hyperglycemia inhibits osteoblastogenesis of rat bone marrow stromal cells via activation of the Notch2 signaling pathway
}

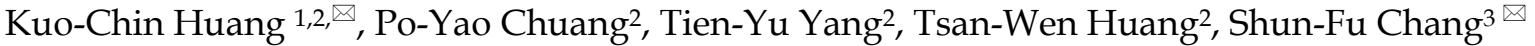 \\ 1. School of Traditional Chinese Medicine, Chang Gung University College of Medicine, Taoyuan City 33302, Taiwan \\ 2. Department of Orthopaedic Surgery, Chiayi Chang Gung Memorial Hospital, Chiayi County 61363, Taiwan \\ 3. Department of Medical Research and Development, Chiayi Chang Gung Memorial Hospital, Chiayi County 61363, Taiwan \\ $\triangle$ Corresponding author: Kuo-Chin Huang, MD, PhD and Shun-Fu Chang, PhD. Department of Orthopaedic Surgery, Chiayi Chang Gung Memorial Hospital, \\ No. 6, West Sec., Chiapu Rd., Putz City, Chiayi County 61363, Taiwan. Tel: (+886)-5-362-1000 ext. 2855. E-mail: kc2672@gmail.com and \\ shunfuchang@cgmh.org.tw \\ (C) Ivyspring International Publisher. This is an open access article distributed under the terms of the Creative Commons Attribution (CC BY-NC) license \\ (https://creativecommons.org/licenses/by-nc/4.0/). See http://ivyspring.com/terms for full terms and conditions.
}

Received: 2019.01.01; Accepted: 2019.03.23; Published: 2019.05.10

\begin{abstract}
Background: Bone fragility and related fractures are increasingly being recognized as an important diabetic complication. Mesenchymal progenitors often serve as an important source of bone formation and regeneration. In the present study, we have evaluated the effects of diabetes on osteoblastogenesis of mesenchymal progenitors.
\end{abstract}

Methods: Primary bone marrow stromal cells (BMSCs) were isolated from control and streptozotocin-induced diabetic rats. These cells were evaluated for the effects of in vivo hyperglycemia on the survival and function of mesenchymal progenitors. We concomitantly investigated the effects of different concentrations of glucose, osmolality, and advanced glycation end product (AGE) on osteogenic differentiation and matrix mineralization of rat bone marrow mesenchymal stem cells (RMSC-bm). The relationship between the expression levels of Notch proteins and the corresponding ALP levels was also examined.

Results: Our results revealed that in vivo hyperglycemia increased cell proliferation rate but decreased osteogenic differentiation and matrix mineralization of primary rat BMSCs. In vitro high glucose treatment, instead of high AGE treatment, induced a dose-dependent inhibition of osteoblastogenesis of RMSC-bm cells. Activation of the Notch2 signaling pathway, instead of the Notchl or osmotic response pathways, was associated with these diabetic effects on osteoblastogenesis of mesenchymal progenitors.

Conclusions: Hyperglycemia might inhibit osteoblastogenesis of mesenchymal progenitors via activation of the Notch2 signaling pathway.

Key words: Diabetes mellitus, Hyperglycemia, Osteoblastogenesis, Mesenchymal Progenitor, Notch signaling pathway

\section{Introduction}

Fragility fractures are being increasingly recognized as an important complication of both type 1 diabetes mellitus (T1DM) and type 2 diabetes mellitus (T2DM), and are associated with excessive morbidity, mortality and health-care costs [1]. The extensive prospective Nurses' Health Study revealed that the incidence of hip fractures in T1DM patients is 2.5-fold higher than that in the T2DM population, and 6-fold higher than that in the general population [2]. There is also a moderate increase in the number of fractures of the spine, distal forearm and proximal humerus in diabetic populations [3]. The pathophysiological mechanisms underlying bone fragility and increased risk of fragility fractures in 
diabetes mellitus (DM) are complex and not completely understood [4]. Some of these mechanisms can potentially alter the fate of mesenchymal progenitors, the initial precursor and major source of osteoblasts, leading to impaired osteogenic differentiation, compromised matrix mineralization, and thus reduced bone strength [5]. Therefore, understanding the diabetes-bone association and studying the process of cell fate determination of mesenchymal progenitors in the diabetic microenvironment is critical for improving bone fragility and decreasing the risk of fragility fractures in patients with DM.

Various signaling factors have been implicated in the regulation of maintenance and expansion of mesenchymal progenitors, including those from the Notch signaling pathway [6]. Notch signaling is an important pathway in that it regulates cell-to-cell signal transduction, which plays an essential role in skeletal remodeling [7]. Notch signaling requires cell-cell contact, and is initiated when the Notch ligands bind to the Notch receptors expressed on the surface of a neighboring cell, then the Notch intracellular domain (NICD) is cleaved and released, followed by its translocation from the cell membrane to the nucleus. In the nucleus, the NICD interacts with the transcriptional regulator of the CSL family to regulate downstream target genes [8]. A study, shows that, NICD overexpression prevents the biological effects of BMP-2 and Wnt by suppressing Wnt but not BMP signaling [9], however, others showed that Notch signaling promotes osteogenic differentiation of mesenchymal progenitors by enhancing BMP signaling [10], whereas, inhibition of Notch signaling impaired BMP2-induced osteoblast differentiation [11]. Besides, Notch1 and Notch2 proteins may function differently and even in reverse manners in the same cell type [12]. Therefore, further studies are necessary to delineate the relationship between Notch and the other signaling pathways under physiological or pathological conditions.

In the present study, we evaluated the effects of diabetes on osteoblastogenesis of mesenchymal progenitors. To address this issue, we investigated the effects of in vivo hyperglycemia, increased glucose or normal glucose levels in vitro, and high osmotic treatments on cellular proliferation, osteogenic differentiation, and matrix mineralization of mesenchymal progenitors. We also assessed the relationship between the Notch expression and osteogenic differentiation in response to treatment with different concentrations of glucose and advanced glycation end product (AGE).

\section{Materials and methods}

\section{Animals and streptozotocin (STZ)-induced diabetic models}

Seven-week-old male Sprague-Dawley (SD) rats were purchased from BioLASCO Taiwan Co., Ltd. (Taipei, Taiwan), and were housed under environmentally controlled conditions with ad libitum access to standard laboratory chow. The Institutional Animal Care and Use Committee (IACUC) of Chang Gung Memorial Hospital approved the animal use protocol, and all animal experiments followed the Animal Protection Law by the Council of Agriculture, Executive Yuan, ROC, and were performed according to the guidelines for the Care and Use of Laboratory Animals as promulgated by the Institute of Laboratory Animal Resources, National Research Council, USA.

Diabetes was induced in SD rats with a single intraperitoneal (IP) administration of STZ $(65 \mathrm{mg} / \mathrm{kg}$ of body weight) diluted in citrate buffer $(0.01 \mathrm{M}, \mathrm{pH}=$ 4.3). Control animals received the buffer alone. Animals were given food and water ad libitum and their body weights were continuously monitored. Blood glucose levels were evaluated at regular intervals using a glucometer (Accu-Check, Roche Diagnostics, Basel, Switzerland), and rats with blood glucose level > $250 \mathrm{mg} / \mathrm{dL}$ were considered to be diabetic. Glycated hemoglobin (HbA1c) levels were concomitantly evaluated using the ARKRAY Automatic Glycohemoglobin Analyzer ADAMS A1c (ARKRAY Factory Inc., Shiga, Japan).

\section{Isolation of primary rat bone marrow MSCs (BMSCs)}

We isolated primary rat bone marrow by cutting both ends of the femur and tibia, and flushing the bone marrow with Dulbecco's modified Eagle's medium (DMEM, Life Technologies Inc., Gaithersburg, MD, USA). After flushing the bone marrow, we filtered the cell suspension through a 70 $\mu \mathrm{m}$ filter to obtain a single cell suspension. Then, we lysed the RBCs using ammonium chloride (at a 9:1 ratio) for $10 \mathrm{~min}$ on ice. After lysing RBCs, we removed ammonium chloride by centrifugation and washing the cell pellet once with culture medium. We then plated the BMSCs isolated from the rats into a T75 flask. The first change of medium was performed less than $72 \mathrm{~h}$ after plating the cells in the flask.

\section{Cell lines and cell cultures}

Rat bone marrow mesenchymal stem cells (RMSC-bm) were obtained from ScienCell Research Laboratory (Carlsbad, CA, USA) and grown in DMEM supplemented with $10 \%$ fetal bovine serum 
(FBS; Life Technologies Inc.) and $1 \%$ penicillin/streptomycin at $37{ }^{\circ} \mathrm{C}$ in a $5 \% \quad \mathrm{CO}_{2}$ humidified incubator. The culture medium was exchanged with a medium that was identical except that it contained only $0.5 \%$ FBS, and the cells were further incubated for $24 \mathrm{~h}$ before treatment with high glucose or normal glucose but high osmotic conditioned media.

\section{Preparation of high glucose and normal glucose but high osmotic conditioned media}

To prepare high glucose conditioned media, we supplemented DMEM (5.5 mM of glucose) with additional glucose at 4 final concentrations of 5.5, 15, 25 , and $35 \mathrm{mM}$ of glucose. To prepare normal glucose but high osmotic conditioned media, we supplemented DMEM (5.5 mM of glucose) with 0, 9.5, 19.5, and $29.5 \mathrm{mM}$ of mannitol, respectively. AGE was purchased from BioVision (Glucose AGE-BSA-II, BioVision Inc., Milpitas, CA, USA). For osteogenic differentiation, cells were cultivated in 6-well plates until $80 \%$ confluence and then incubated in the medium containing ascorbate, $ß$-glycerophosphoate, and dexamethasone (Sigma-Aldrich $\mathrm{GmbH}$, Schnelldorf, Germany).

\section{Analysis of cell proliferation}

Cell proliferation was analyzed using XTT assays (Biological Industries, Kibbutz Beit-Haemek, Israel), for which the cells were plated in 96-well plates and allowed to adhere for $24 \mathrm{~h}$. Culture medium was changed every 3 days. An ELISA plate reader (Thermo Labsystems Multiskan RC, Vantaa, Finland) was used to measure absorbance of samples at a wavelength of $490 \mathrm{~nm}$.

\section{Western blotting}

Cultured/treated cells were lysed with the lysis buffer containing protease and phosphatase inhibitors (phenylmethylsulfonylfluoride, aprotinin, and sodium orthovanadate). Total protein from the cell lysate (100 $\mu \mathrm{g}$ of protein) was separated using SDS-PAGE (using a 10\% running, and $4 \%$ stacking polyacrylamide gel) and separated proteins were transferred onto a nitrocellulose membrane (Immobilon $\mathrm{P} ; 0.45-\mu \mathrm{m}$ pore size). The blot was then treated with the indicated antibodies. Chemiluminescent bands were detected by using the Western-Light chemiluminescent detection system (Applied Biosystems). COL1 and ALP expression levels were normalized with those of $B$-actin internal control from the same sample.

\section{ARS quantification assay}

We used Alizarin Red-S (ARS) staining to study the effects of high glucose treatment on matrix mineralization. After treatment for 14 days, culture medium was removed and the cells were fixed with $70 \%$ ice-cold ethanol (v/v) for $10 \mathrm{~min}$ and rinsed thoroughly with distilled water. Cultures were then stained with $40 \mathrm{mM}$ ARS in deionized water ( $\mathrm{pH}$ 4.2) for $10 \mathrm{~min}$ at room temperature. After removing the ARS solution by aspiration, cells were rinsed with fresh PBS and dried at room temperature. ARS concentrations were then calculated by comparison using an ARS dye standard curve and expressed as $\mathrm{nM} / \mathrm{mL}$.

\section{Statistical analysis}

Results were expressed as means \pm SEM. One-way analysis of variance (ANOVA) with Scheffe's post hoc test was performed and Spearman's rank correlation coefficient $\left(\mathrm{r}_{\mathrm{s}}\right)$ was calculated using SPSS v13.0 (SPSS, Chicago, IL, USA). $P<0.05$ was considered as statistically significant.

\section{Results}

\section{Effects of in vivo hyperglycemia on cell} proliferation, osteogenic differentiation and matrix mineralization of primary rat BMSCs

Administration of STZ successfully induced diabetes in all rats, as revealed by increased mean blood glucose at the time of sacrifice $(349.3 \pm 16.1$ $\mathrm{mg} / \mathrm{dL}$ vs. $101.8 \pm 3.9 \mathrm{mg} / \mathrm{dL}$ in control rats, $P<0.001$ ) (Fig. 1A). HbA1c levels were elevated accordingly $(9.18 \pm 0.12 \%$ vs. $4.20 \pm 0.09 \%$, respectively, $P<0.001)$ (Fig. 1B).

To determine whether in vivo hyperglycemia impairs survival and functions of mesenchymal progenitors, we harvested primary BMSCs from control and STZ-induced diabetic rats. As determined using the XTT assay, our data revealed that primary BMSCs from the STZ-induced diabetic rats had a higher proliferation rate than that of BMSCs from the control rats (1.54 fold on day $7, P<0.05)$ (Fig. 1C). On the other hand, these cells showed decreased osteogenic differentiation and matrix mineralization, as seen using western blotting ( $31 \%$ and $42 \%$ decrease in COL1 and ALP expression, respectively, $P<0.05$ for both) (Fig. 1D) and ARS staining quantification assay $(0.35 \pm 0.03$ vs. $0.41 \pm 0.01, P=0.121)$ (Fig. 1E). These results suggest that in vivo hyperglycemia in STZ-induced diabetic rats is associated with an increased proliferation rate but decreased osteogenic differentiation and matrix mineralization of their mesenchymal progenitors.

\section{Effects of different concentrations of glucose on osteogenic differentiation and matrix mineralization of RMSC-bm cells}

To examine the effects of different 
A

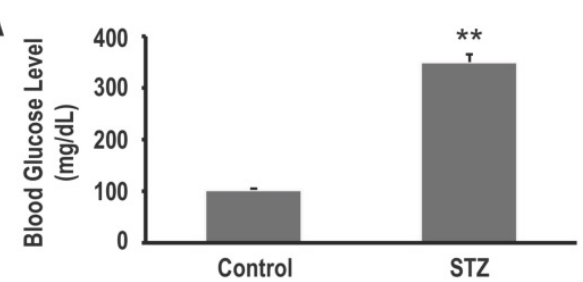

C

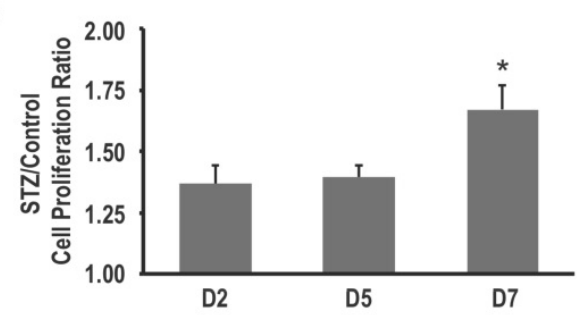

E

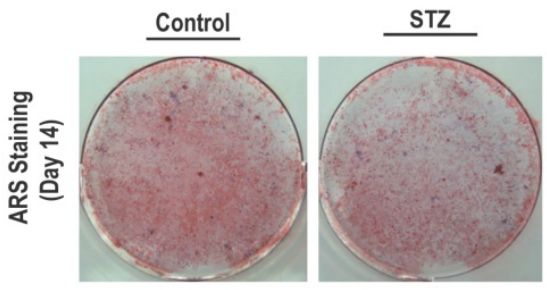

B

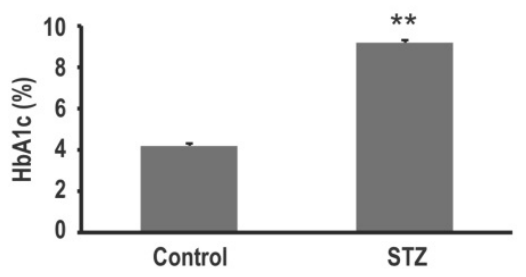

D
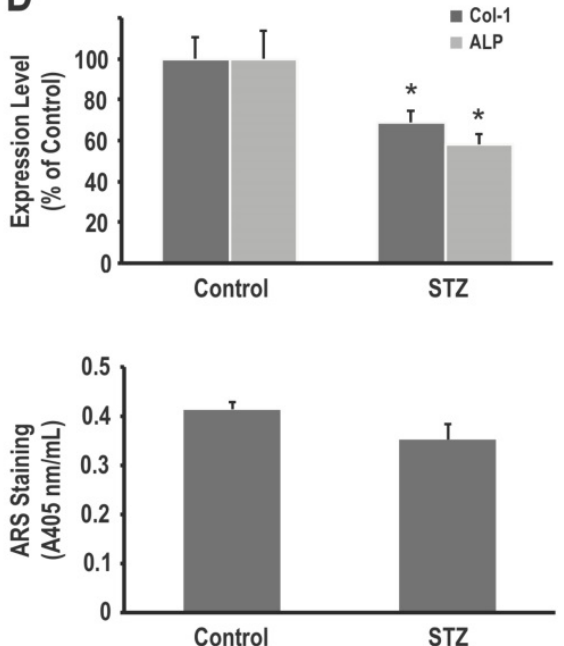

Figure 1. Hyperglycemia in STZ-induced diabetic rats is associated with an increased proliferation rate but decreased osteogenic differentiation and matrix mineralization of primary bone marrow stromal cells (BMSCs). (A) Blood glucose levels and (B) HbAlc levels of normoglycemic (control) and diabetic (STZ) rats. Primary BMSCs from control and diabetic rats were harvested, and their cell proliferation rates were determined using the XTT assay (C), COL1 and ALP expression levels in BMSCs from control and diabetic rats were determined using western blotting (D), and matrix mineralization in BMSCs was determined using the ARS staining quantification assay $(\mathrm{E})$. Data are represented as mean \pm SEM from three to four independent experiments. $*, P<0.05$ vs. control cells. $* *, P<0.001$ vs. control cells.

concentrations of glucose on osteogenic differentiation and matrix mineralization, we used RMSC-bm cells in culture as a model system. As determined using western blotting, high glucose (25 $\mathrm{nM} / \mathrm{L})$ treatment for 7 days inhibited expression of both COL1 and ALP (27\% and 36\% decrease, respectively, $P<0.05$ for both) (Fig. 2A). As determined using the ARS staining quantification assay, high glucose treatment for 14 days induced dose-dependent inhibition of calcium deposition $(P<$ 0.05) (Fig. 2B). These results suggested that high glucose treatment could lead to a deleterious impact on osteogenic differentiation and matrix mineralization of mesenchymal progenitors.

\section{Effects of the osmotic effect of glucose on osteogenic differentiation of RMSC-bm cells}

To determine the role of osmotic effect of glucose in osteogenic differentiation, we cultured RMSC-bm cells in different concentrations of high glucose and normal glucose but high osmotic conditioned media. As determined using western blotting, our data revealed that there were no significant differences in either COL1 or ALP expression among treatments with different concentrations of normal glucose but high osmotic conditioned media. On the contrary, high glucose treatment for 7 days induced dose-dependent inhibition of COL1 and ALP expression in RMSC-bm cells (Fig. 3). These results suggest that the deleterious effects of high glucose treatment on osteogenic differentiation could not be ascribed to the osmotic effect of glucose.

\section{Effects of different concentrations of glucose and AGE on Notch expression and osteogenic differentiation of RMSC-bm cells}

Because of the recent insights into the role of Notch signaling in regulating bone physiology and in causing human bone diseases [7], we examined the relationship between Notch expression and high glucose-induced inhibition of osteogenic differentiation. As determined using western blotting, our data revealed that high glucose treatment for both 2 and 7 days would induce dose-dependent increase in Notch2 expression in RMSC-bm cells. However, there were no significant differences in Notch1 expression among treatments with different concentrations of glucose. Meanwhile, Notch2 expression levels showed a moderate to strong negative association with corresponding ALP expression levels $\left(r_{\mathrm{s}}=-0.674, P<0.05\right)$ in response to 

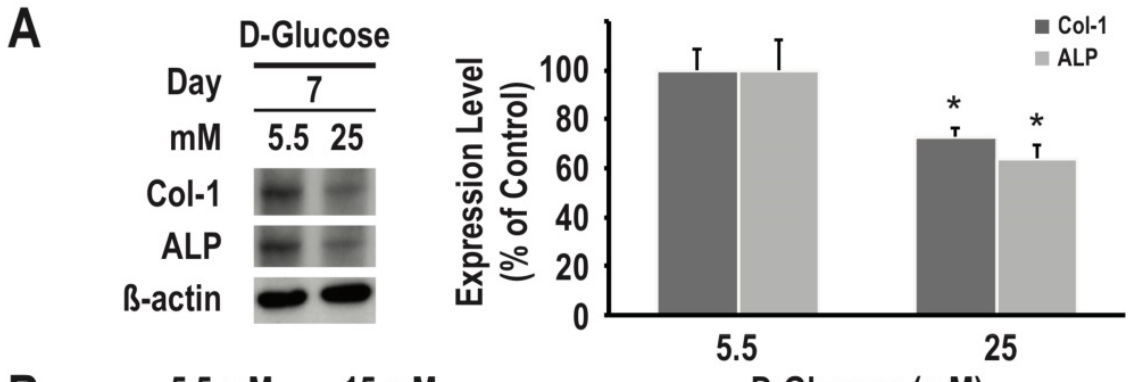

B
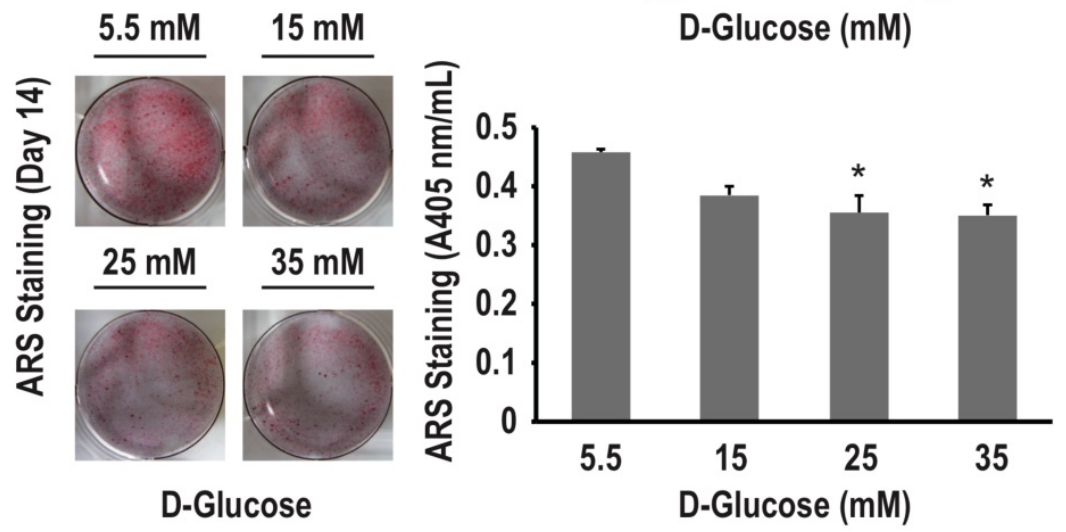

Figure 2. High glucose treatment presents a dose-dependent inhibitory effect on osteogenic differentiation and matrix mineralization of mesenchymal progenitors. RMSC-bm cells were used as control $(5.5 \mathrm{mM})$, or stimulated with high glucose $(15,25$, and $35 \mathrm{mM})$ for 7 and 14 days. Western blotting was used to determine COL1 and ALP expression levels in RMSC-bm cells (A), $\beta$-actin used as an internal control. The ARS staining quantification assay was used to define the extent of matrix mineralization in RMSC-bm cells (B). Data are represented as mean \pm SEM from three to four independent experiments. * $P<0.05$ vs. control cells.

treatments with different concentrations of glucose (Fig. 4A). We further investigated the effects of different concentrations of AGE on Notch expression and osteogenic differentiation of RMSC-bm cells as the gradual increase or accumulation of AGE is considered to be an important cause of diabetic complications. Our data revealed that AGE treatment results in non-significant changes of Notch2 and ALP expression. Besides, Notch2 expression levels did not indicate an association with the corresponding ALP expression levels $\left(\mathrm{r}_{\mathrm{s}}=0.487, P=0.11\right)$ in response to treatments with different concentrations of AGE (Fig. $4 \mathrm{~B})$. These results suggest that activation of the Notch2 signaling pathway might play a role in high glucose-induced inhibition of osteoblastogenesis of mesenchymal progenitors.

\section{Discussion}

This study suggests the potential mechanism underlying the effects of diabetes on osteoblastogenesis of mesenchymal progenitors. Our findings revealed that in vivo hyperglycemia in STZ-induced diabetic rats is associated with an increased cell proliferation rate; however, shows decreased osteogenic differentiation of primary BMSCs. High glucose treatment presents a dose-dependently inhibitory effect on osteogenic differentiation and matrix mineralization of RMSC-bm cells. The deleterious effects of high glucose treatment on osteogenic differentiation could not be ascribed to the osmotic effect of glucose. On the other hand, activation of the Notch2, instead of the Notch1, signaling pathway might play a critical role in high glucose-induced suppression of osteoblastogenesis and subsequent compromised osteogenic differentiation and reduced matrix mineralization. Our findings provide insights into the molecular mechanisms by which DM affects cell fate determination of mesenchymal progenitors. Given that Notch signaling affects cell fate decision of BMSCs and that the effects are dependent on the cellular context, it may be critical to develop therapeutic options that can preferentially target the osteoblastic lineage cells of diabetic patients with bone fragility and related fragility fractures.

$\mathrm{DM}$ is characterized by hyperglycemia. Some in vitro studies have indicated that hyperglycemia can be toxic to osteoblasts [4]. Botolin et al. have shown that hyperglycemia and its associated hyperosmolality suppress expression of genes involved in osteoblast maturation [13]. Cunha et al. further demonstrated that MC3T3-E1 osteoblasts respond to high extracellular glucose concentrations through an osmotic response pathway that results in modulation of ALP and OCN expression and uptake of calcium by osteoblasts in culture [14]. Contrastingly, mesenchymal progenitors are more resistant to the toxicity caused by hyperglycemia than osteoblasts, 
depending on the stemness of mesenchymal progenitors [15]. This is supported by our findings that primary BMSCs from the STZ-induced diabetic rats have an increased proliferation rate as compared to that of BMSCs from control rats. Some studies showed that hyperglycemia and oxidative stress might influence differentiation of mesenchymal progenitors with adipogenesis being favored over osteoblastogenesis [16]. In agreement with an earlier observation in mesenchymal progenitors from STZ-induced diabetic rats [17], we found that both, in vivo hyperglycemia and in vitro high glucose treatment, affect osteoblastogenesis of mesenchymal progenitors. Our data demonstrates that the harmful effect of hyperglycemia in mesenchymal progenitors is independent of the extracellular osmolality but is most likely attributed to the impact on cell fate decision of mesenchymal progenitors. Indeed and surprisingly, Notch2 expression levels presented a moderate to strong negative association with the corresponding ALP expression levels $\left(\mathrm{r}_{\mathrm{s}}=-0.674, P<\right.$ 0.05 ) in response to treatments with different concentrations of glucose.

$\mathrm{DM}$ is also characterized by accumulation of AGEs. Levels of AGEs are increased in DM patients as a result of chronic hyperglycemia and increased levels of oxidative stress [18], and might play a crucial role in the development of bone fragility and related fragility fractures in these patients [5]. Yamamoto et al. indicated that serum AGE (pentosidine) levels are positively associated with the presence of vertebral fractures in postmenopausal Japanese women with T2DM [19]. Saito et al. found that the degree of mineralization correlates with distinctive patterns of enzymatic and non-enzymatic cross-links in human bone [20]. Kume et al. showed that AGEs might lead to in vivo loss of MSC mass and to the delay of bone repair by inhibiting the maturation of MSC-derived cells [21]. Taken together, accumulation of AGEs in the organic bone matrix by the Maillard reaction should be negatively associated with biomechanical, dynamic, and microarchitectural skeletal properties [22]. These data, although limited, would suggest that AGE-distorted collagen likely renders the bone more fragile in DM patients regardless of their bone mineral density (BMD). In the current study, our data did not support the idea that AGEs per se directly affect osteoblastogenesis of mesenchymal progenitors. We found that the AGE-BSA treatment did not result in significantly enhanced ALP expression in RMSC-bm cells, which would metabolize calcium phosphate into insoluble phosphate salts, thereby mediating matrix mineralization [23]. As the effects of AGEs presumably vary according to the source of AGEs, the type of cells, and culture conditions, further studies will be required to clarify the details of the mechanism of AGE-mediated control of MSC osteoblastogenesis, particularly studies modeling the in vivo microenvironment for the processes of diabetic complications.

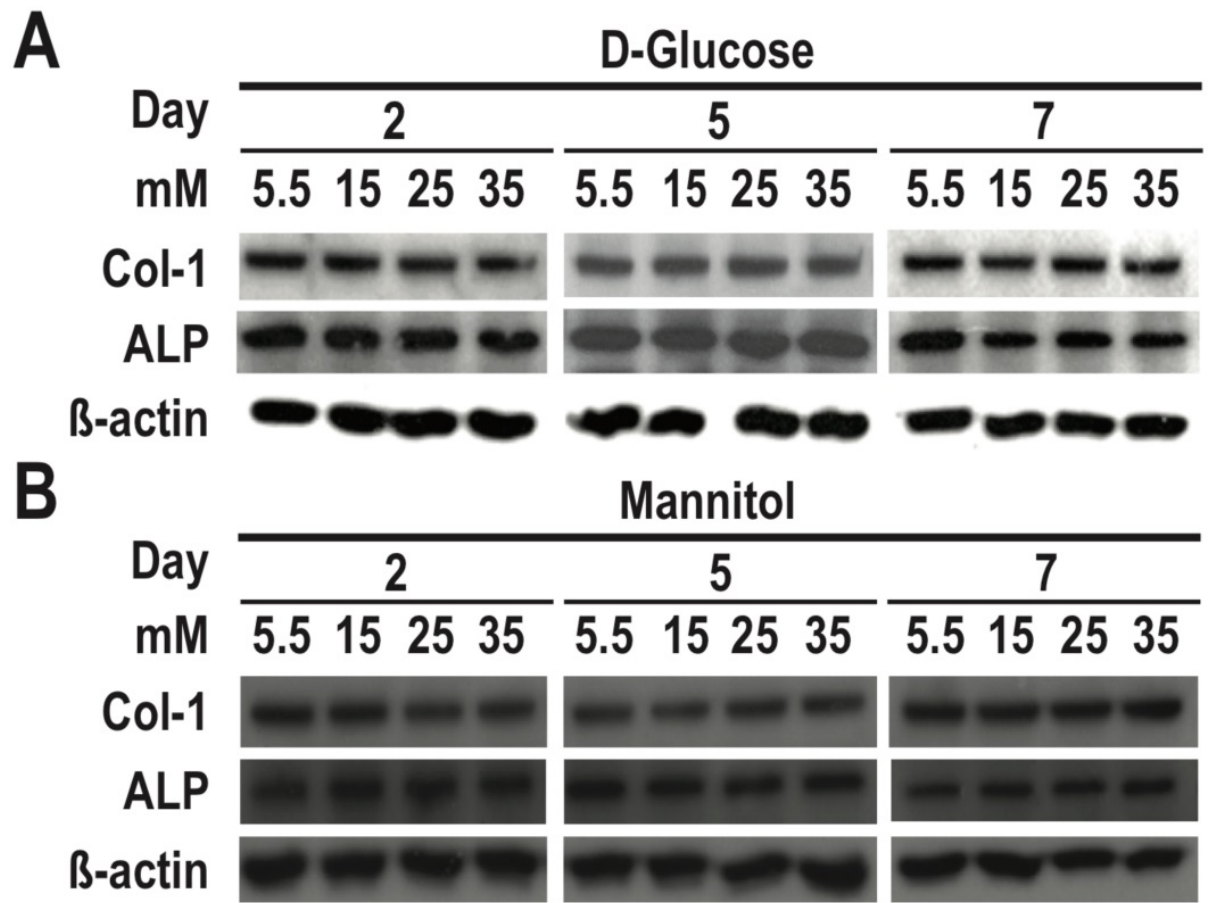

Figure 3. The deleterious effects of high glucose treatment on osteogenic differentiation could not be ascribed to the osmotic effect of glucose. RMSC-bm cells were used as control $(5.5 \mathrm{mM})$, or stimulated with (A) high glucose $(15,25$, and $35 \mathrm{mM})$, and (B) normal glucose but high osmotic $(5.5 \mathrm{mM}$ glucose $+9.5 \mathrm{mM}$ mannitol, 5.5 $\mathrm{mM}$ glucose $+19.5 \mathrm{mM}$ mannitol, and $5.5 \mathrm{mM}$ glucose $+29.5 \mathrm{mM}$ mannitol) for 2, 5, and 7 days. Western blotting was used to determine COLI and ALP expression levels in RMSC-bm cells while $\beta$-actin used as an internal control. 
A

Day $\frac{2}{\text { mM }} \frac{2}{5.5152535} \frac{7}{5.5152535}$

Notch 1

Notch 2 - $---\cdots$

B-actin
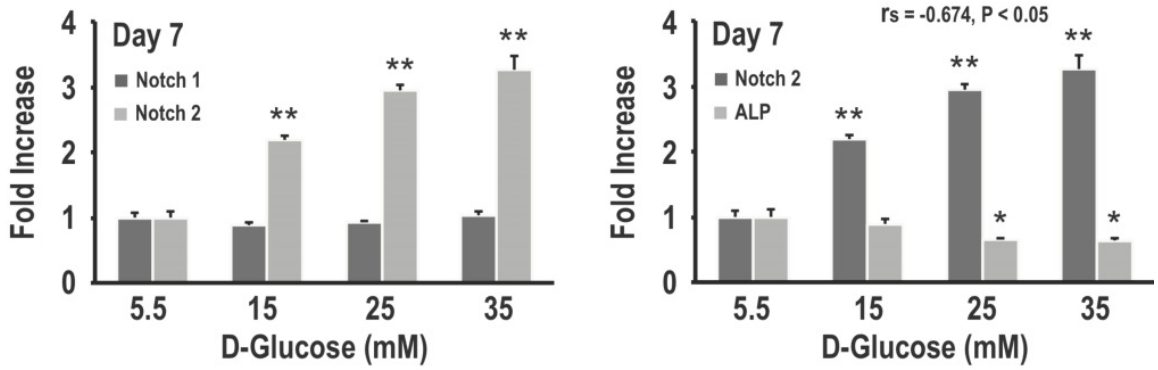

B

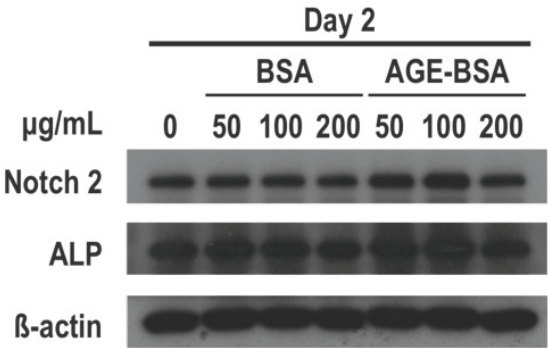

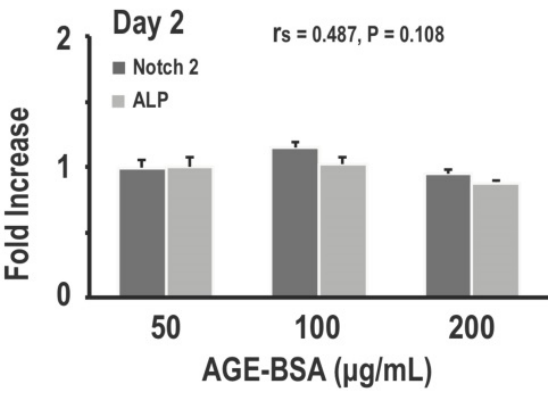

Figure 4. Activation of the Notch2 signaling pathway might play a role in high glucose-induced inhibition of osteogenic differentiation. RMSC-bm cells were used as control (5.5 mM), or stimulated with high glucose $(15,25$, and $35 \mathrm{mM})$ for 2 and 7 days. Western blotting was used to determine Notch1, Notch2, and ALP expression levels in RMSC-bm cells (A), $\beta$-actin used as an internal control. RMSC-bm cells were used as control (50 $\mu \mathrm{g} / \mathrm{mL})$, or stimulated with high AGE (100 and $200 \mu \mathrm{g} / \mathrm{mL})$ for 2 days. Western blotting was used to determine Notch 2 and ALP expression levels $(B), \beta$-actin used as an internal control. Data are represented as mean \pm SEM from three to four independent experiments. $r_{s}$, Spearman's rank correlation coefficient. $*, P<0.05$ vs. control cells. $* *, P<0.001$ vs. control cells.

Our results revealed that Notch2, instead of Notch1, plays a role in high glucose-induced inhibition of osteoblastogenesis of mesenchymal progenitors. Notch signaling, discovered in Drosophila, is well known for its role in cell fate decisions and the development of multiple tissues including bone [24]. Notch signaling may interact with other signaling pathways such as BMP and Wnt, the two critical factors known to enhance osteoblastogenesis $[25,26]$, to regulate bone homeostasis [7]. Although many studies reported that Notch signaling inhibits osteoblastogenesis of mesenchymal progenitors $[9,27,28]$, some other studies demonstrated that Notch sensitizes cells of the osteoblastic lineage to the effects of inducers of osteoblastogenesis under selective conditions $[10,11,29]$. Generally, Notch might be required not for the function of mature osteoblasts, but for the differentiation of their mesenchymal precursors [28]. Engin et al. further indicated that the conditional deletion of notch2 would cause a similar developmental phenotype to the dual deletion of notch 1 and notch2, which suggests that notch 2 might be the predominant regulator of endochondral bone formation [27]. Our data contribute to the debate regarding the role of Notch signaling in osteoblastogenesis of mesenchymal progenitors, supporting that the Notch2, instead of the Notch1, signaling pathway might play a critical role in diabetic bone fragility and related fragility fractures.

\section{Conclusions}

Hyperglycemia, instead of AGE, inhibits osteoblastogenesis of mesenchymal progenitors, which might be through activation of the Notch2, instead of the Notch1 or osmotic response, signaling pathway. This fact suggests the possibility of a new therapeutic strategy to treat various diabetic complications including bone fragility and related fragility fractures. As a molecule that might selectively inhibit osteoblastogenesis of mesenchymal progenitors, Notch2 may be expected to be a unique target molecule for the treatment of diabetic complications such as bone fragility and related fragility fractures.

\section{Acknowledgement}

This work was supported by grants from the 
Chang Gung Memorial Hospital, Taiwan (Grant Nos. CMRPG 6C0081-3, and CMRPG 6G0061) and from the Taiwan National Science Council (Grant No. NSC102-2314-B-182A-033).

\section{Competing Interests}

The authors have declared that no competing interest exists.

\section{References}

1. Janghorbani M, Van Dam RM, Willett WC, Hu FB. Systematic review of type 1 and type 2 diabetes mellitus and risk of fracture. Am J Epidemiol. 2007; 166(5): 495-505.

2. Janghorbani M, Feskanich D, Willett WC, Hu FB. Prospective study of diabetes and risk of hip fracture: the Nurses' Health Study. Diabetes Care. 2006; 29(7): 1573-1578.

3. Vestergaard P, Rejnmark L, Mosekilde L. Relative fracture risk in patients with diabetes mellitus, and the impact of insulin and oral antidiabetic medication on relative fracture risk. Diabetologia. 2005; 48(7): 1292-1299.

4. Napoli N, Strollo R, Paladini A, Briganti SI, Pozzilli P, Epstein S. The alliance of mesenchymal stem cells, bone and diabetes. Int J Endocrinol. 2014; 2014: 690783.

5. Napoli N, Chandran M, Pierroz DD, Abrahamsen B, Schwartz AV, Ferrari SL. Mechanisms of diabetes mellitus-induced bone fragility. Nat Rev Endocrinol. 2017; 13(4): 208-219.

6. Dong $\mathrm{Y}$, Long $\mathrm{T}$, Wang $\mathrm{C}$, Mirando AJ, Chen J, O'Keefe RJ, et al. $\mathrm{NOTCH}$-mediated maintenance and expansion of human bone marrow stromal/stem cells: a technology designed for orthopedic regenerative medicine. Stem Cells Transl Med. 2014; 3(12): 1456-1466.

7. Regan J, Long F. Notch signaling and bone remodeling. Curr Osteoporos Rep. 2013; 11(2): 126-129.

8. Canalis E. Notch signaling in osteoblasts. Sci Signal. 2008; 1(17): pe17.

9. Deregowski V, Gazzerro E, Priest L, Rydziel S, Canalis E. Notch 1 overexpression inhibits osteoblastogenesis by suppressing Wnt/ $\mathrm{B}$-catenin but not bone morphogenetic protein signaling. J Biol Chem. 2006; 281(10): 6203-6210.

10. Cao J, Wei Y, Lian J, Yang L, Zhang X, Xie J, et al. Notch signaling pathway promotes osteogenic differentiation of mesenchymal stem cells by enhancing BMP9/Smad signaling. Int J Mol Med. 2017; 40(2): 378-388.

11. Nobta M, Tsukazaki T, Shibata Y, Xin C, Moriishi T, Sakano S, et al. Critical regulation of bone morphogenetic protein-induced osteoblastic differentiation by Delta1/Jagged1-activated Notch1 signaling. J Biol Chem. 2005; 280(16): 15842-15848.

12. Sun Y, Gao X, Liu J, Kong QY, Wang XW, Chen XY, et al. Differential Notch1 and Notch2 expression and frequent activation of Notch signaling in gastric cancers. Arch Pathol Lab Med. 2011; 135(4): 451-458.

13. Botolin S, Faugere $M$, Malluche $H$, Orth $M$, Meyer R, McCabe LR. Increased bone adiposity and peroxisomal proliferator-activated receptor- $\gamma 2$ expression in type I diabetic mice. Endocrinology. 2005; 146(8): 3622-3631.

14. Cunha JS, Ferreira VM, Maquigussa E, Naves MA, Boim MA. Effects of high glucose and high insulin concentrations on osteoblast function in vitro. Cell Tissue Res. 2014; 358(1): 249-256.

15. Li YM, Schilling T, Benisch P, Zeck S, Meissner-Weigl J, Schneider D, et al. Effects of high glucose on mesenchymal stem cell proliferation and differentiation. Biochem Biophys Res Commun. 2007; 363(1): 209-215.

16. Aguiari P, Leo S, Zavan B, Vindigni V, Rimessi A, Bianchi K, et al. High glucose induces adipogenic differentiation of muscle-derived stem cells. Proc Natl Acad Sci USA. 2008; 105(4): 1226-1231.

17. Weinberg E, Maymon T, Moses O, Weinreb. Streptozotocin-induced diabetes in rats diminishes the size of the osteoprogenitor pool in bone marrow. Diabetes Res Clin Pract. 2014; 103(1): 35-41.

18. Piperi C, Adamopoulos C, Dalagiorgou G, Diamanti-Kandarakis E, Papavassiliou AG. Crosstalk between advanced glycation and endoplasmic reticulum stress: emerging therapeutic targeting for metabolic diseases. J Clin Endocrinol Metab. 2012; 97(7): 2231-2242.

19. Yamamoto $M$, Yamaguchi T, Yamauchi M, Yano S, Sugimoto T. Serum pentosidine levels are positively associated with the presence of vertebral fractures in postmenopausal women with type 2 diabetes. J Clin Endocrinol Metab. 2008; 93(3): 1013-1019.

20. Saito M, Fujii K, Marumo K. Degree of mineralization-related collagen crosslinking in the femoral neck cancellous bone in cases of hip fracture and controls. Calcif Tissue Int. 2006; 79(3): 160-168.

21. Kume S, Kato S, Yamagishi S, Inagaki Y, Ueda S, Arima N, et al. Advanced glycation end-products attenuate human mesenchymal stem cells and prevent cognate differentiation into adipose tissue, cartilage, and bone. J Bone Miner Res. 2005; 20(9): 1647-1658.

22. Leslie WD, Rubin MR, Schwartz AN, Kanis JA. Type 2 diabetes and bone. J Bone Mine Res. 2012; 27(11): 2231-2237.
23. Beck GR Jr, Sullivan EC, Moran E, Zerler B. Relationship between alkaline phosphatase levels, osteopontin expression, and mineralization in differentiating MC3T3-E1 osteoblasts. J Cell Biochem. 1998; 68(2): 269-280.

24. Bugeon L, Taylor HB, Progatzky F, Lin MI, Ellis CD, Welsh N, et al. The $\mathrm{NOTCH}$ pathway contributes to cell fate decision in myelopoiesis. Haematologica. 2011; 96(12): 1753-1760.

25. Gazzerro E, Canalis E. Bone morphogenetic proteins and their antagonists. Rev Endocr Metab Disord. 2006; 7(1-2): 51-65.

26. Krishnan V, Bryant HU, MacDougald OA. Regulation of bone mass by Wnt signaling. J Clin Invest. 2006; 116(5): 1202-1209.

27. Engin F, Yao Z, Yang T, Zhou G, Bertin T, Jiang MM, et al. Dimorphic effects of Notch signaling in bone homeostasis. Nat Med. 2008; 14(3): 299-305.

28. Hilton MJ, Tu X, Wu X, Bai S, Zhao H, Kobayashi T, et al. Notch signaling maintains bone marrow mesenchymal progenitors by suppressing osteoblast differentiation. Nat Med. 2008; 14(3): 306-314.

29. Tezuka K, Yasuda M, Watanabe N, Morimura N, Kuroda K, Miyatani S, et al. Stimulation of osteoblastic cell differentiation by Notch. J Bone Miner Res. 2002; 17(2): 231-239. 